\section{Influence of HLA-A, HLA-B, and HLA-DR matching on rejection of random corneal grafts using corneal tissue for retrospective DNA HLA typing}

\author{
Marjolijn C Bartels, Henderikus G Otten, B Elske van Gelderen, Allegonda Van der Lelij
}

\begin{abstract}
Department of Ophthalmology, University Hospital Rotterdam, Netherlands M C Bartels

Department of Medical Immunology, University Medical Centre Utrecht, Netherlands H G Otten

Department of Ophthalmo-Immunology, The Netherlands Ophthalmic Research

Results-A prolonged rejection free survival time was observed in graft recipients with one or two HLA-A matches (log rank test, $p=0.034)$. This effect was also observed in high risk graft recipients with one or two HLA-DR matches (log rank test, $\mathrm{p}=\mathbf{0 . 0 3 0}$ ).

Conclusions-Coincidental HLA-A and HLA-DR matches were observed and associated with a prolonged rejection free survival time in the total group and in the high risk group, respectively. These results support the beneficial effect of prospective HLA-A and HLA-DR typing upon corneal graft survival.

(Br f Ophthalmol 2001;85:1341-1346)
\end{abstract} Institute, Amsterdam, Netherlands

B E van Gelderen

Department of Ophthalmology, Leiden University Medical Centre,

Netherlands

A Van der Lelij

Correspondence to: Dr M C Bartels, Department of Ophthalmology, University Hospital Rotterdam, PO Box 2040, 3000 CA Rotterdam, Netherlands

bartels@oogk.azr.nl

Accepted for publication 23 May 2001

Corneal grafting is often a successful procedure. ${ }^{1}$ In contrast with transplantation of other solid organs, no systemic immunosuppressive agents are needed to prevent allograft rejection. ${ }^{2}$ In avascular corneas, graft survival results of $85-95 \%$ were reported after 1 year of follow up. ${ }^{13}$ Nevertheless, immunological allograft rejection is reported to be the leading cause of corneal graft failure. ${ }^{34}$ The incidence of immune mediated graft rejection and failure is increased in high risk patients having corneal vascularisation or a history of graft rejection..$^{5-8}$ It might be expected that corneal graft survival in high risk cases would increase after prospective human leucocyte antigen (HLA) class I matching of donor and recipient, ${ }^{5-12}$ in

\begin{abstract}
Aim-To establish if coincidental HLA-A, HLA-B, and HLA-DR tissue matching is associated with a reduced likelihood of corneal graft rejection.

Methods-Organ culture preserved random donor corneas were used for penetrating keratoplasty (PKP). Corneal tissue from all graft recipients and donors or blood samples from recipients after repeated transplantation were obtained in order to perform retrospective molecular HLA typing. A group of 21 recipients with a rejection episode (cases) after corneal transplantation was compared with a control group of non-rejectors $(n=43) .31$ graft recipients were considered as high risk patients. The influence of HLA-A, HLA-B, and HLA-DR matching on rejection free graft survival time was analysed with Kaplan-Meyer statistics and Cox regression.
\end{abstract}

accordance with HLA matching for increased graft survival of other solid organs. ${ }^{13}{ }^{14}$ However, the role of HLA matching in reducing corneal graft failure could not be confirmed by all studies. ${ }^{3}{ }^{15-18}$ A beneficial role of HLA-A and HLA-B matching can be explained by the finding that mismatched HLA class I antigens are immunogenic. ${ }^{19}{ }^{20}$ HLA class I antigens are targets for rejection by $\mathrm{CD} 8+$ cytotoxic $\mathrm{T}$ lymphocytes. ${ }^{21}$

Organ culture preservation of donor corneas was shown to contribute to a reduction in the amount of HLA class II bearing Langerhans cells, ${ }^{22-24}$ resulting in reduced antigenicity of the corneal graft. ${ }^{1925}$ This may lead to a decreased incidence of rejection. HLA-DR (class II) matching in cornea transplantation showed beneficial effects ${ }^{26}$ and an adverse effect in another study. ${ }^{28}$ This controversy may be explained partly by inadequacy of serological HLA-DR typing techniques. ${ }^{29}$

Although HLA matched corneal grafts were rarely used in patients at high risk of rejection at the University Medical Centre of Utrecht, Netherlands, a relatively high success rate of corneal transplants was observed over the last 5 years. ${ }^{30}$ With the recent availability of DNA based typing techniques ${ }^{29} 3132$ it is possible to perform HLA-A, HLA-B, and HLA-DR typing of recipients and donors retrospectively and accurately. ${ }^{33-36}$ The aim of this retrospective study was to examine whether differences exist with regard to molecular HLA-A, HLA-B, and HLA-DR matching and to rule out coincidental HLA matching between corneal graft recipients, with versus without immune mediated graft rejection.

\section{Patients and methods} PATIENTS

Between February 1995 and December 1997 248 consecutive patients underwent PKP with random corneal grafts at the University Medical Centre of Utrecht, Netherlands. Patient charts were revised for age, sex, use of systemic immune suppression, a history of glaucoma or herpes simplex keratitis (HSK), tear film insufficiency, number of previous grafts, indication for transplantation, degree of stromal vascularisation of the recipient cornea at the time of PKP, additional surgical procedures during PKP, any rejection episodes, and the final outcome of the corneal graft. In the category of patients with a history of glaucoma we included patients with ocular hypertension or primary open angle glaucoma (POAG) based 
Table 1 Clinical characteristics of recipients and donors from the case and control group

\begin{tabular}{lll}
\hline & Cases & Controls \\
\hline Number & 21 & 43 \\
Age (years): mean (range) & $57.2(29-79)$ & $51.6(11-83)$ \\
Male:female ratio & $1.0: 1.1$ & $1.0: 1.0$ \\
Use of systemic immune suppression & 1 & 1 \\
History of ocular hypertension or POAG & $6(28.6 \%)$ & $9(20.9 \%)$ \\
History of HSK & $7(33.3 \%)$ & $14(32.6 \%)$ \\
Tear film insufficiency & $2(9,5 \%)$ & $3(7.0 \%)$ \\
Number of previous grafts: & $15(71.4 \%)$ & $35(81.4 \%)$ \\
0 & $4(19.0 \%)$ & $8(18.6 \%)$ \\
1 & $2(9.5 \%)$ & $0(0 \%)$ \\
$\quad>1$ & $11(52.4 \%)$ & $15(34.9 \%)$ \\
Vascularisation of recipient cornea & & \\
Additional procedures: & $3(14.3 \%)$ & $9(20.9 \%)$ \\
$\quad$ Cataract extraction & $3(14.3 \%)$ & $4(9.3 \%)$ \\
Synechiolysis & $1(4.8 \%)$ & $2(4.7 \%)$ \\
$\quad$ Iris reconstruction & $69.0(20-89)$ & $67.8(16-86)$ \\
Donor age (years): mean (range) & $1.7: 1.0$ & $1.9: 1.0$ \\
Donor sex; male:female ratio & $15.8(7-23)$ & $15.9(10-27)$ \\
Time in culture medium (days): mean (range) & $7.5(6.5-8.0)$ & $7.5(5.5-8.0)$ \\
Graft size (mm): mean (range) & & \\
\hline
\end{tabular}

on use of antiglaucoma medication, neuropathy with an excavation of the optical nerve, defects in the visual field, a diagnosis of POAG, or an intraocular pressure above $23 \mathrm{~mm} \mathrm{Hg}$. Furthermore, information about the donors and donor corneas was collected; age, sex, graft size, and storage time of organ culture preservation. High risk criteria for corneal allograft failure were defined according to the presence of two or more quadrants of deep stromal vascularisation and/or previous immunologically mediated allograft failure (s). ${ }^{9}$

Out of the group of 248 patients a case $(n=$ $21)$ and control group $(n=43)$ were selected. Cases were all transplant recipients undergoing a rejection episode, according to strict criteria described below $(n=23)$. Patients for whom it was not possible to differentiate between irreversible graft rejection and graft failure due to other causes were not included. Patients in whom no corneal tissue was available for DNA isolation ( $\mathrm{n}=2$, one patient grafted for Fuchs' dystrophy and the other for HSK) were excluded. The control group was formed out of a group of patients with at least 1 year of follow up after PKP and no episodes of graft rejection. This group was matched for age and sex. Patients with graft failure because of other causes than rejection were not excluded as long as their cornea could be evaluated for rejection during the study period. Eight out of 43 grafts failed irreversibly as a result of other causes than graft rejection, two grafts failed as a consequence of intractable glaucoma, and six because of presumed slow endothelial decompensation.

In the group of cases, 12 out of $21(57 \%)$ could be considered as high risk and in the group of controls 19 out of 43 (44\%).

Patient, donor, and graft characteristics of cases and controls are presented in Table 1. No significant differences were found between the two groups with respect to these characteristics. The indications for PKP are shown in Table 2 .

DIAGNOSIS OF REJECTION

The time between PKP and the first rejection episode, not necessarily ending in graft failure, was used to analyse graft outcome. The
Table 2 Indications for PKP of cases and controls

\begin{tabular}{lcc}
\hline Indication for PKP & Cases & Controls \\
\hline HSK & 7 & 12 \\
Allograft failure & 6 & 8 \\
Keratoconus & - & 11 \\
Pseudophakic BKP & 2 & 5 \\
Corneal ulcers & 4 & 3 \\
Trauma & 2 & 2 \\
Fuchs' dystrophy & - & 2 \\
\hline
\end{tabular}

diagnosis of immune mediated allograft rejection was based on generally accepted criteria. ${ }^{37}$ All corneal grafts had to become clear after surgery and a diagnosis of rejection was not made for at least 10 days after surgery. Rejection episodes were not diagnosed during a period of elevated ocular pressure. The presence of a "Khodadoust line" was considered a pathognomonic sign of graft rejection. In the absence of a Khodadoust rejection line, graft rejection was diagnosed by the presence of cells in the anterior chamber, in combination with an increase in (partial) corneal thickness and/or keratic precipitates limited to the graft. For patients with a history of HSK, graft rejection, and recurrence of herpes simplex virus (HSV) infection needed to be differentiated. If no Khodadoust line was observed, additional tests were needed to diagnose HSV infection. Aqueous humour was obtained by paracentesis of the anterior chamber to determine intraocular antibody production against HSV, expressed as the Goldmann-Witmer coefficient for HSV. Also, PCR analysis of HSV DNA was performed $^{38}$ to exclude an active ocular herpes simplex infection. Herpes infected cases were excluded from analysis in this study.

\section{METHODS}

Donor procurement and medical procedures did not change during the study period. All donor corneas were obtained from Bio Implant Service and the Dutch Eye Bank, Amsterdam, Netherlands. The donor corneas were organ culture preserved as described by Pels et al. ${ }^{39}$ All donor corneas were stored in organ culture of Eagle's minimum essential medium for at least 7 days with a maximum of 27 days. PKP procedures were performed by one of three ophthalmic surgeons using similar surgical techniques. All patients were treated with a combination of prednisolone eye ointment $(0.5 \%$, at bedtime) and local dexamethasone/ gentamicin eye drops $(0.1 \%$, six times daily) after surgery, for 4 weeks. At least 1-1.5 years after PKP, dexamethasone eye drops were applied and gradually tapered off. If PKP was combined with a cataract extraction or removal of an intraocular lens, additional eye drops were given, containing a non-steroid antiinflammatory drug $(0.1 \%$, four times daily) during the first 3 months after surgery. Rejection reactions were treated with hourly applications of prednisolone acetate $1 \%$ eye drops and three periocular injections with $0.1 \%$ dexamethasone, given every other day. Graft rejection has led to irreversible graft failure in nine out of 21 cases. 
DNA TYPING FOR HLA

Corneal tissue was used for retrospective DNA

HLA typing. For this purpose, corneal tissue of the recipients and corneal rims of donor corneas were collected at the time of surgery and stored frozen (at $-80^{\circ} \mathrm{C}$ ). After repeated transplantation blood samples containing peripheral blood mononuclear cells were taken from recipients to obtain DNA. Corneal tissues and blood derived cell suspensions were subjected to DNA extraction using the Qiagen DNA extraction kit (Boehringer, Mannheim, Germany) for blood and tissues, according to the manufacturer's instructions. DNA solutions were stored at $-80^{\circ} \mathrm{C}$ until further amplification. The diagnosis of rejection at the end of the follow up was made before the HLA typing was known. HLA typing was done without knowledge of graft outcome. This molecular typing of HLA-A, HLA-B, and HLA-DRB1 (all low resolution) was performed according to a reverse hybridisation line probe assay as described by the manufacturer of InnoLiPa (Innogenetics, Gent, Belgium). ${ }^{40}$

Typing data were available in all 64 donor/ recipient pairs for HLA-DR. For HLA-A and HLA-B one and five pairs were not available respectively, owing to shortness of donor DNA $(\mathrm{n}=2)$, technical problems with PCR $(\mathrm{n}=1)$, and problems with analysing the Lipa test results for HLA-B $(\mathrm{n}=3)$. Analysis was performed both on the broad HLA typing level (A1, A2, A3, A9, A10, A11, A19, A28, B5, B7, $\mathrm{B} 8, \mathrm{~B} 12, \mathrm{~B} 13, \mathrm{~B} 14, \mathrm{~B} 15, \mathrm{~B} 16, \mathrm{~B} 17, \mathrm{~B} 18, \mathrm{~B} 21$, B22, B27, B35, B37, B40, B41, B42, B53, B70, DR1，DR2，DR3，DR4，DR5，DR6，DR7, DR8, DR9, DR10) and split typing level (splits from A9, A10, A19, A28, B5, B12, B16, B17, B21, B22, B70, DR2, DR3, DR5, DR6).

ANALYSIS AND STATISTICS

Comparability of patient, donor, and graft covariates between cases and controls were analysed using the $\chi^{2}$ test for categorical variables and the independent $T$ test for continuous covariates. To analyse rejection free graft survival, cases and controls were subdivided into a group with and a group without matched HLA subtypes. The group with matches consisted of cases and controls with one or two matches of HLA-A, HLA-B, or HLA-DR. Unadjusted survival probabilities for the total group as well as the high and low risk group were estimated using Kaplan-Meier statistics and differences were assessed using the log rank test. ${ }^{41}$ All tests were two sided and a $p$ value of less than 0.05 was taken as statistically significant.

To assess the clinical relevance of matching we used Cox regression analysis estimating the odds ratios (OR) and 95\% confidence intervals for the association between HLA matching per HLA locus and duration of rejection free graft survival, adjusting for confounding variables. Covariates were chosen for inclusion in the model if they were mildly correlated $(p<0.20)$ with rejection according to univariate analysis. Using this selection criterion, only vascularisation of the recipient cornea was included in the model $(\mathrm{p}=0.12)$.
Table 3 Degree of HLA-A, HLA-B, and HLA-DR matching of the case and control group

\begin{tabular}{llcc}
\hline & $\begin{array}{l}\text { No of split } \\
\text { matches }\end{array}$ & Cases & Controls \\
\hline HLA-A & 0 & $16(76 \%)$ & $21(50 \%)$ \\
& 1 & $4(19 \%)$ & $15(36 \%)$ \\
HLA-B & 2 & $1(5 \%)$ & $6(14 \%)$ \\
& 0 & $14(74 \%)$ & $30(75 \%)$ \\
HLA-DR & 1 & $5(26 \%)$ & $9(22.5 \%)$ \\
& 2 & 0 & $1(2.5 \%)$ \\
& 0 & $14(67 \%)$ & $25(58 \%)$ \\
& 1 & $6(29 \%)$ & $17(40 \%)$ \\
& 2 & $1(5 \%)$ & $1(2 \%)$ \\
\hline
\end{tabular}

\section{Results}

The median follow up period of the control group ( $n=43$ ) was 2.6 years (range $1-4$ years). The median time until rejection of the case group ( $\mathrm{n}=21$ ) was 9 months (range 12 days to 1 year and 11 months).

The maximum number of matches based on split typing for a patient in this study was three, which was detected in six controls and one case. The distribution of HLA-A, HLA-B, and HLA-DR split matches in cases and controls is shown in Table 3. Recalculation of split HLA DNA typing results into broad typing results did not yield significant differences between cases and controls with regard to HLA-A and HLA-B matching. However, an increased amount of broad HLA-DR matches was found compared to the amount of split HLA-DR matches in four cases.

Corneal graft recipient-donor pairs with HLA-A matches had a longer rejection free graft survival compared with corneal grafts without HLA-A matches (log rank test, $\mathrm{p}=$ 0.034, Fig 1). We observed a trend towards a prolonged rejection free graft survival with increasing numbers of HLA-A matches. No difference was observed in rejection free graft survival time between cases and controls with respect to HLA-B matches (Fig 2) and HLA-DR matches (Fig 3).

Analysing rejection free graft survival for the high risk $(n=31)$ and low risk $(n=33)$ group separately, a stronger association between longer rejection free graft survival and HLA-A matching in the low risk group was found (log rank test, $p=0.013)$. Graft survival was not significantly longer with respect to HLA-B matches in the high or low risk group (data not

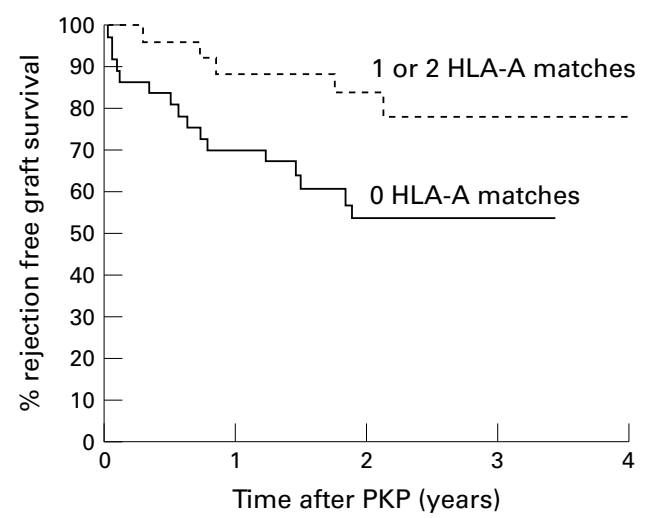

Figure 1 Kaplan-Meier curves indicating rejection free survival of corneal graft recipients with one or two matches at the HLA-A locus is significantly better than those with 0 $H L A-A$ matches (log rank test, $p=0.030, n=63$ ). 


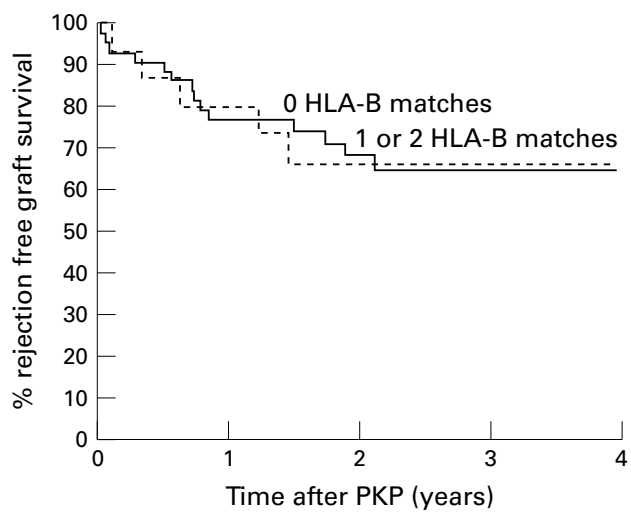

Figure 2 Kaplan-Meier curves indicating the presence of $H L A-B$ matches did not improve rejection free survival of corneal graft recipients (log rank test, $p=0.50, n=59$ ).

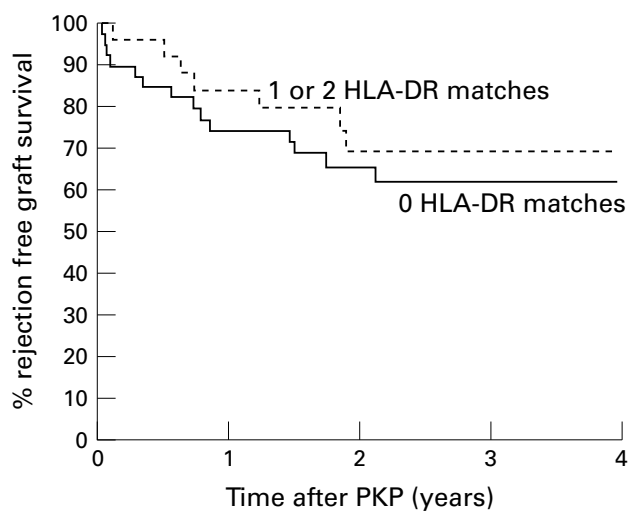

Figure 3 Kaplan-Meier curves indicating the presence of $H L A-D R$ matches did not improve rejection free survival of corneal graft recipients (log rank test, $p=0.92, n=64$ ).

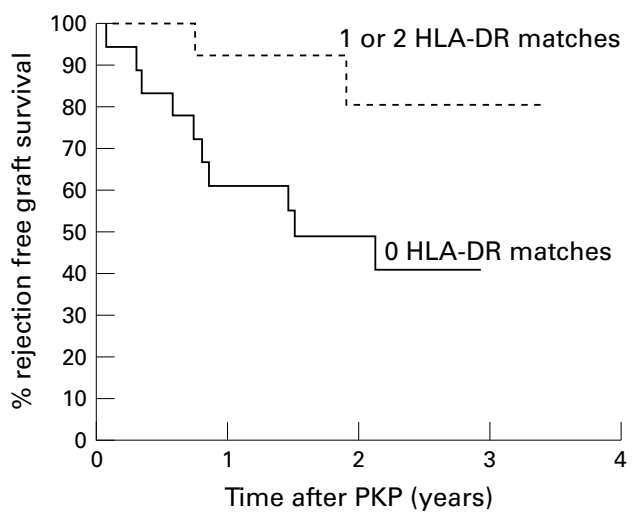

Figure 4 Kaplan-Meier curves indicating HLA-DR matching in high risk recipients showed a beneficial effect on the rejection free graft survival (log rank test, $p=0.030$, $n=31$ ).

shown). Analysis of the HLA-A and HLA-B matches together showed no effect in the total group or in the high risk group. However, we observed a beneficial effect of matching for HLA-DR in the high risk group (log rank test, $\mathrm{p}=0.030$, Fig 4$)$.

According to Cox regression analysis, after adjustment for vascularisation of the recipient cornea, HLA-A matched transplants (one or two HLA-A matches) were almost three times less likely to reject than HLA-A unmatched transplants (Table 4). HLA-DR matched transplants were slightly, but not significantly, less likely to reject (Table 4).
Table 4 Odds ratios of corneal graft rejection

\begin{tabular}{ll}
\hline & $O R^{\star}(95 \% \mathrm{CI})$ \\
\hline $\begin{array}{l}\text { HLA-A unmatched transplants } \\
\text { HLA-A matched transplants† }\end{array}$ & $0.34(0.13-0.95)$ \\
$\begin{array}{l}\text { HLA-B unmatched transplants } \\
\text { HLA-B matched transplants } \ddagger\end{array}$ & $1.07(0.38-2.97)$ \\
$\begin{array}{l}\text { HLA-DR unmatched transplants } \\
\text { HLA-DR matched transplants } \\
end{array}$ & $0.69(0.27-1.70)$ \\
\hline
\end{tabular}

*Adjusted for vascularisation of the recipient cornea.

tOne or two matches for HLA-A.

$\ddagger$ One or two matches for HLA-B.

SOne or two matches for HLA-DR.

\section{Discussion}

The results showed that the presence of HLA-A matches was associated with prolonged rejection free graft survival. When considering patients at high risk for rejection, a beneficial effect of HLA-DR matches was shown. These HLA matches were found retrospectively in corneal graft recipients who received random donor corneas at the time of PKP.

Cases with and controls without an immune mediated graft rejection were analysed together according to their rejection free graft survival time. The first rejection episode, not necessarily leading to graft failure, was used as the end point of analysis. From an immunological point of view it is more appropriate to use rejection free graft survival time rather than time to immunological graft failure. Whether a rejection episode will lead to graft failure is dependent on treatment protocols of a rejection reaction used in different transplantation centres. Furthermore, each rejection episode accelerates the postoperative loss of endothelial cells, resulting in earlier graft failure. ${ }^{42}{ }^{43}$

A major advantage of HLA DNA typing, as used in this study, is that this method is less prone to errors than serological typing techniques. $^{31}{ }^{32}$ Discrepancy rates of $10-25 \%$ have been reported for serological HLA-DR typing in kidney transplantation. ${ }^{29}$ DNA can be derived from any organic tissue, including corneal tissue, which is easily obtained at $\mathrm{PKP}^{44}$ and nearly always available in contrast with blood samples necessary for serological typing.

The diagnosis of an immune mediated graft rejection is not always clearcut. Rejection and recurrence of HSK can be impossible to distinguish clinically and may coexist. ${ }^{45}$ We excluded patients for whom it was not possible to differentiate between graft rejection and HSK.

Patient compliance is of great influence on graft survival. ${ }^{6}$ In our study topical steroids were given for at least 1 year in all patients. All patients with graft rejection within 1 year were still using topical steroids. These data, however, are based on patient records and a contribution of patient compliance to graft rejection could not be fully excluded.

HLA-A and HLA-B antigens have been identified on corneal epithelium, stromal cells, and corneal endothelial cells. ${ }^{19}{ }^{46} 47$ The HLA class I antigens are targets for CD8+ cytotoxic $\mathrm{T}$ cells in the process of graft rejection. ${ }^{21}$ Theoretically, matching for HLA-A and HLA-B therefore would reduce the amount of target 
antigens, which could lead to a decreased incidence of rejection. An accumulating number of authors suggested that HLA-A and HLA-B matching was associated with improved outcome of corneal graft survival in high risk recipients. ${ }^{5-12} 15334849$ These data on HLA-A matching are in line with the results from our study. However, other studies, including the large Collaborative Corneal Transplantation Study, failed to demonstrate a correlation of HLA matches and graft survival. ${ }^{16-18} 50$ A beneficial HLA-A matching effect was observed in all risk categories with a stronger association in low risk cases by Boisjoly et al, ${ }^{11}$ which is in accordance with our findings. In this study, HLA-B matches showed no significant effects on graft rejection. This might be due to the overall low number of matches observed in this study at the polymorphic HLA-B locus. In previous studies, matching of HLA-B showed a beneficial effect on corneal graft survival $^{510} 12$ and an adverse effect in others. ${ }^{18} 20$

Despite the fact that HLA-DR is considered to be important in renal transplantation, ${ }^{29}$ the role of HLA-DR matching in corneal transplantation remains controversial. ${ }^{49} 51$ Significant beneficial effects of HLA-DR matching in high risk recipients were reported using RFLP typing techniques, ${ }^{26}$ but a historical control group was used. Japanese studies $^{33} 3444$ using DNA typing techniques reported a strong beneficial effect of HLA-DP matching and no effect of matching for HLA-DR. This may be explained by the overall low number of HLA-DR matches reported by this group, using donors and recipients from different ethnic backgrounds. In our study, both donors and recipients originated mostly from the white population, and therefore from the same gene pool. This may add to the explanation of the high number of coincidental HLA-A and HLA-DR matches we observed. In some studies, a beneficial influence of HLA-DR matching could not be found in low or high risk recipients, ${ }^{16} 1752$ whereas in other studies an adverse effect was reported. ${ }^{28} 51$ However, in these studies serological typing techniques were used, which in general yield accurate broad but not split typing results. In our study HLA DNA typing included analysis of HLA split antigens. A beneficial effect of HLA-DR matching in high risk recipients was shown. After conversion of HLA-DR split to broad antigens (data not shown), a significant effect of matching could no longer be found, which is in line with the previously mentioned studies.

Expression of HLA-DR antigens carried on Langerhans cells has been demonstrated within normal corneal epithelium and stromal layers. ${ }^{19} 47$ Migration and accumulation of Langerhans cells in the central part of the cornea, which is normally devoid of Langerhans cells $^{19}{ }^{24}$ can be established by vascularisation and inflammation..$^{53}$ As a consequence of an increased number of Langerhans cells in the centre of the donor cornea, a rejection reaction may be provoked. ${ }^{54}$ The beneficial effect of HLA-DR matching for high risk recipients as reported in our study might be explained by a higher number of Langerhans cells in the central area of the cornea after transplantation in this high risk group.

A drawback of this study is the overall low number of included patients. Owing to the random distribution of corneal grafts a relatively small number of patients with more than one match at HLA-A, HLA-B, or HLA-DR loci was included. As a consequence it was not possible to evaluate combinations of HLA-A and HLA-B matches or to evaluate the effect of HLA-DR in the absence of any HLA-A or HLA-B match.

Although the case and control group are comparable with respect to the characteristics mentioned in Table 1, the indications for transplantation were not completely comparable, with Fuchs' dystrophy $(\mathrm{n}=2)$ and keratoconus $(n=11)$ being present only in the control group. One of the two excluded cases (no donor corneal tissue available) was transplanted for Fuchs' dystrophy; if this patient had been included, the only remaining difference would have been the indication of keratoconus. Analysis of rejection free graft survival without considering these 11 patients showed the same results for the high risk group (a longer rejection free graft survival for HLA-DR matched transplants). Furthermore, rejection free graft survival was longer for HLA-A matched transplants in the total group $(n=52)$, despite the loss of power $(p=0.047)$.

We included controls with at least 1 year of follow up. However, rejections are still observed after 1 year. Therefore, further studies with a longer follow up time will be needed to show a beneficial long term outcome with matching.

Organ culture preservation of donor corneas, as used for all donor corneas in this study, allows time to allocate corneas based on HLA matching. Prospective DNA HLA typing and transportation of donor corneas over long distances to the most suitable recipient could give logistical problems if short term preservation techniques, such as McCarey-Kaufman medium, are used. The cost-benefit ratio of providing matched corneal grafts should be investigated in more detail. Especially in more heterogeneous populations where less coincidental HLA matches are expected, allocation of matched donor corneas could be more difficult but also more effective in preventing corneal graft rejection.

In conclusion, molecular typing of HLA-A, HLA-B, and HLA-DRB1 is an accurate method and offers the possibility to perform HLA typing at any time postoperatively, using corneal derived DNA. Although all corneal graft recipients received random donor corneas, retrospectively a significantly higher number of HLA-A matches was found, in favour of recipients with longer rejection free graft survival. Furthermore, a beneficial effect of matching for HLA-DR was shown for high risk recipients. These results add to the evidence that prospective molecular HLA-A and HLA-DR typing has a beneficial effect upon graft survival. 
1 Williams KA, Lewis RF, Coster DJ. How successful is corneal transplantation? A report from the Australian corneal graft register. Eye 1995;9:219-27.

2 Vail A, Gore SM, Bradley BA, et al. Corneal graft survival and visual outcome, a multicenter study. Ophthalmology

3 Vail A, Gore SM, Bradley BA, et al. Conclusions of the corneal transplant follow up study. Br f Ophthalmol 1997;81: 631-6.

4 Khodadoust AA. The allograft rejection reaction: the leading cause of late failure of clinical corneal grafts. In Corneal graft failure. Ciba Foundation symposium. Amsterdam: Elsevier 1973:151-67.

5 Völker-Dieben HJ, Kok-van Alphen CC, Lansbergen Q, et al. The effect of prospective HLA-A and -B matching on corneal graft survival. Acta Ophthalmol 1982;60:203-12.

6 Williams KA, Roder D, Esterman A, et al. Factors predictive of corneal graft survival. Ophthalmology 1992;99:403-44.

7 Boisjoly HM, Tourigny R, Bazir R, et al. Risk factors of corneal graft failure. Ophthalmology 1993;11:1728-35.

8 Völker-Dieben HJ, Kok-van Alphen CC, Lansbergen O, et Völker-Dieben HJ, Kok-van Alphen CC, Lansbergen Q, et
al. Different influence on corneal graft survival in 539 al. Different influence on corneal graft survival

9 Foulks GN, Sanfillipo F. Beneficial effects of histocompatibility in high-risk corneal transplantation. Am $\mathcal{f}$ Ophthalmol 1982;94:622-9.

10 Sanfilippo F, MacQueen JM, Vaughn WK, et al. Reduced graft rejection with good HLA-A and B matching in high-

1 Boisjoly HM, Roy R, Bernard P-M, et al. Association between corneal allograft rejections and HLA compatibility. Ophthalmology 1990;12:1689-98.

12 Ozdemir O. A prospective study of histocompatibility testing for keratoplasty in high risk patients. $\mathrm{Br} \mathcal{F} O$ phthalmol 1986;70:183-6.

13 Dyer PA, Claas FH. A future for HLA matching in clinical transplantation. Eur f Immunogenet 1997;24:17-28.

14 Friend PJ. Rejection reactions to different organ transplants. Eye 1995;9:190-219.

15 Batchelor JR, Casey TA, Gibbs DC, et al. HLA matching and corneal grafting. Lancet 1976;1:551-4.

16 The Collaborative Corneal Transplantation Studies. Effectiveness of histocompatibility matching in high risk corneal transplantation. Arch Ophthalmol 1992;110:1392-403.

17 Fink N, Stark WJ, Maguire MG, et al. Effectiveness of histocompatibility matching in high-risk corneal transplantation. A summary of results from the Collaborative Corneal Transplantation Studies. Cesk Oftalmol 1994;50:3-12.

18 Hill JC, Creemers PC. An adverse matching effect for the HLA-B locus in corneal transplantation. Transplant Int 1997;10:145-9.

19 Pels E, Van der Gaag R. HLA-A, B, C and HLA-DR antigens and dendritic cells in fresh and organ cultured preserved corneas. Cornea 1985:3:231-9.

20 Creemers PC, Kahn D, Hill JC. HLA-A and -B alleles in cornea donors as risk factors for graft rejection. Transplant Immunol 1999;7:15-18.

21 Roelen DL, van Beelen E, van Bree SP, et al. The presence of activated donor HLA class I-reactive T lymphocytes is associated with rejection of corneal grafts. Transplantation associated with rejec

22 Pels E, Schuchard Y. Organ culture in the Netherlands, preservation and endothelial evaluation. In: Brightbill FS, ed. Corneal surgery. 2nd ed. St Louis: Mosby, 1986:622-31

23 Armitage WJ. The effects of storage of corneal tissue on Langerhans cells. Eye 1995;9:228-32.

24 Ardjomand N, Komericki P, Radner $\mathrm{H}$, et al. Korneale Langerhans-Zellen verhalten während der Lagerung in Organkultur. Der Ophthalmol 1997;10:703-6.

25 Niederkorn JY. The immune privilege of corneal allografts. Transplantation 1999;67:1503-8.

26 Baggesen K, Ehlers N, Lamm LU. HLA-DR/RFLP compatible corneal grafts. Acta Ophthalmol 1991;69:22933.

27 Baggesen K, Lamm LU, Ehlers N. Significant effect of highresolution HLA-DRB1 matching in high-risk corneal resolution HLA-DRB1 matching in high-risk

28 Bradley BA, Vail A, Gore SM, et al. Negative effect of HLA-DR matching on corneal transplant rejection. Transplant Proc 1995;1:1392-4.

29 Mytilineos J, Scherer S, Hansen B, et al. RFLP-DR beta and serological HLA-DR typing of 200 kidney recipients and 1000 controls. Transplant Proc 1990;4:1911-12.
30 Gelderen van BE, Van der Gaag R, Pels E, et al. Analysis of graft failure with random, organ cultured, donor corneas in low and high risk patients. A single centre study. (Submitted for publication.)

31 Middleton D, Savage DA, Culler C, et al. Discrepancies in serological typing revealed by DNA techniques. Transplant Int 1988;1:161-4.

32 Bidwell J. Advances in DNA-based HLA-typing methods. Immunol Today 1994;7:303-7.

33 Morita N, Munkhbat B, Kanai N, et al. Effect of HLA-A and -DPB1 matching in corneal transplantation. Transplant Proc 1998;30:3491-2.

34 Munkhbat B, Hagihara M, Sato $\mathrm{T}$, et al. Association between HLA-DPB1 matching and 1-year rejection-free graft survival in high-risk corneal transplantation. Transplantation 1997;7:1011-16.

35 Thonnard J, Deldome F, Heusterspreute M, et al. HLA class II genotyping: two systems compared. Clin Chem 1995;41: 553-6.

36 Stuyver L, Rossau R, Wyseur A, et al. Typing of hepatitis C virus isolates and characterization of new subtypes using a line probe assay. F Gen Virol 1993;74:1093-102.

37 Ciba Foundation Symposium. Criteria for a diagnosis of a corneal allograft rejection. In: Corneal graft failure. Ciba Foundation Symposium 15. Amsterdam: Elsevier, 1973: $340-7$.

38 Boer de JH, Luyendijk L, Rothova A, et al. Detection of intraocular antibody production to herpesviruses in acute retinal necrosis syndrome. Am f Ophthalmol 1994;117:20110.

39 Pels E, Schuchard Y. Organ-culture preservation of human corneas. Doc Ophthalmol 1983;56:147-53.

40 Puchhammer-Stockl E, Schmied B, Mandl CW, et al. Comparison of line probe assay (LIPA) and sequence analysis for detection of HIV-1 drug resistance. 7 Med Virol 1999;57:283-9.

41 Schouten HJA, Clinical Statistics. [Een praktische inleiding in methodologie en analyse, Dutch.] Kaplan Meyer analysis, Data management, Chi quadrate, Logistic regression analysis. Bohn Stafleu Van Loghum, Houten/Diegem, Netherlands 1995:101-8, 128-98.

42 Ing JJ, Ing $\mathrm{HH}$, Nelson LR, et al. Ten-year postoperative results of penetrating keratoplasty. Ophthalmology 1998; 105:1855-65.

43 Nishimura JK, Hodge DO, Bourne WM Initial endothelial cell density and chronic endothelial cell loss rate in corneal transplants with late endothelial failure. Ophthalmology 1999;106:1962-5.

44 Munkhbath B, Hagihara M, Sato T, et al. HLA Class II DNA typing using ocular tissue and its usefulness in corneal transplantation. Transplant Proc 1996;3:1257-8.

45 Larkin DFP. Corneal transplantation for herpes simplex keratitis. Commentary. Br f Ophthalmol 1998;82:107-8.

46 Whitsett CF, Stulting RD. The distribution of HLA antigen on human corneal tissue. Invest Ophthalmol Vis Sci 1984:25:519-24.

$47 \mathrm{Li} \mathrm{Q}, \mathrm{He}$ Y. An immunohistochemical study of Langerhans cells, T-cells and the HLA antigen in human cornea. Yen Ko Hsueh Pao 1993;19:121-5.

48 Munkhbat B, Hagihara M, Shimazaki J, et al. The impact of HLA-A matching in corneal transplantation. Tokai f Exp Clin Med 1999;24:63-71.

49 Vail A, Gore SM, Bradley BA, et al. Influence of donor and histocompatibility factors on corneal graft outcome. Trans-

plantation 1994;58:1210-16.
50 Ducrey NM, Glauser MP, Frei PC. Corneal transplantation: ABO blood groups and HLA compatibil-
trans ity. Ann Ophthalmol 1980;7:880-4.

51 Gore SM, Vail A, Bradley BA, et al, on behalf of the corneal transplant follow-up study collaborators. HLA-DR matching in corneal transplantation. Systematic review of published evidence. Transplantation 1995;60:1033-9.

52 Völker-Dieben HJ, D'Amaro J, Kok-van Alphen CC. Hierarchy of prognostic factors for corneal allograft survival. Austr N Z 7 Ophthalmol 1987;15:11-18.

53 Jager MJ. Corneal Langerhans cells and ocular immunology. Reg Immunol 1992;4:186-95.

54 Niederkorn JY. Effect of cytokine-induced migration of Langerhans cells on corneal allograft survival. Eye 1995;9: 215-18. 